\title{
Doença de Parkinson: uma perspectiva neurofisiológica
}

\author{
Parkinson's disease: a neurophysiological perspective
}

\section{Enfermedad de Parkinson: una perspectiva neurofisiológica}

\author{
Carina Gonçalves Pedroso Uchida ${ }^{1}$, Rodrigo Mendes Bakerolov르, Carla \\ Alessandra Scorza ${ }^{1}$
}

1.Departamento de Neurologia e Neurocirurgia, Escola Paulista de Medicina, Universidade Federal de São Paulo (UNIFESP), São Paulo-SP, Brasil.

\section{Resumo}

A doença de Parkinson (DP) é uma condição neurodegenerativa muito prevalente na população idosa, e o diagnóstico é essencialmente clínico, com a melhora dos sintomas motores pela reposição de levodopa. Embora a alteração do funcionamento da alça córtex-núcleos da base (NB) causada pela depleção de dopamina seja o principal mecanismo fisiopatogênico, tanto a fisiologia normal quanto as alterações que ocorrem na DP nestas estruturas ainda não foram totalmente esclarecidas. A estimulação cerebral profunda (deep brain stimulation, DBS) nos NB como modalidade de tratamento alternativa tem ampliado as possibilidades de estudo neurofisiológico da atividade neural, que em associação com os avanços tecnológicos na obtenção, processamento e integração dos sinais córtico-subcorticais, pode trazer novos entendimentos quanto à fisiopatogênese da DP, e inovações terapêuticas.

Unitermos. Doença de Parkinson; neurofisiologia; núcleos da base; estimulação cerebral profunda (DBS)

\begin{abstract}
Parkinson's disease (PD) is a progressive disorder with high prevalence in elderly individuals, diagnosis is based on clinical data, as well as the improvement of motor symptoms with levodopa replacement. Although the impaired function of the cortex-basal ganglia (BG) loop caused by dopamine depletion is the main pathophysiological mechanism, both the normal physiology and the changes related to PD in these anatomical substracts have not been clarified. The deep brain stimulation (DBS) as an alternative treatment in PD has expanded the possibilities of neural activity assessment by neurophysiology, which, associated with technological advances in obtaining, processing, and integrating cortical-subcortical signals, may improve the knowledge about the pathophysiology of PD, as well as enable therapeutic innovations.
\end{abstract}

Keywords. Parkinson Disease; neurophysiology; basal ganglia; deep brain stimulation (DBS)

\begin{abstract}
Resumen
La enfermedad de Parkinson (EP) es un transtorno progressivo muy prevalente en la población anciana, el diagnóstico se basa en los hallazgos clínicos y los sintomas motores mejoran con el reemplazo de levodopa. Aunque la función alterada del bucle corteza-ganglios basales (GB) causada por la depleción de dopamina es el principal mecanismo fisiopatológico, no se han aclarado ni la fisiología normal ni los cambios relacionados com la EP em estos sustratos. La estimulación cerebral profunda (ECP) como tratamiento alternativo em la EP ha ampliado las posibilidades de evaluación de la actividad neural por neurofisiología, que, asociada a los avances tecnológicos en la obtención, procesamiento e integración de señales corticalsubcorticales, puede mejorar el conocimiento sobre la fisiopatología de la EP, además de posibilitar innovaciones terapéuticas.
\end{abstract}

Palabras clave. Enfermedad de Parkinson; neurofisiología; ganglios basales; estimulación cerebral profunda (ECP) 
Trabalho desenvolvido para o curso "Neurociência em Pauta" ("Neuroscience at hand"), ministrado pelo Programa de Pós-Graduação em Neurologia/Neurociências da Escola Paulista de Medicina, Unifesp, São Paulo-SP, Brasil.

\section{INTRODUÇÃO}

A doença de Parkinson (DP) é considerada um grupo de condições neurodegenerativas caracterizada por perda primária dos neurônios dopaminérgicos na substância negra. Os sintomas cardinais são tremor de repouso das mãos, em contar moedas, bradicinesia, rigidez plástica dos membros, e distúrbios da marcha e do equilíbrio. A prevalência estimada em países industrializados é de aproximadamente 0,3\% na população geral, $1 \%$ nas pessoas acima dos 60 anos, e 3,0\% naqueles acima dos 80 anos, com incidência estimada entre 8 e 18 por cem mil/ano. Há uma variedade mais rara chamada DP de início precoce, de forma que 5$10 \%$ dos pacientes apresentam sintomas antes dos 40 anos $^{1}$. A fase pré-motora é caracterizada por distúrbios comportamentais do sono REM, alterações do humor, apatia, ansiedade, constipação e perda do olfato ${ }^{2}$. A DP é heterogênea e pode apresentar formas de progressão mais rápidas e mais lentas ${ }^{3}$. A perda cognitiva e a demência são comuns em fases avançadas, associadas à piora funcional e ao aumento da mortalidade ${ }^{2}$.

A causa da DP é provavelmente multifatorial, resultando de uma interação complexa entre fatores 
ambientais e genéticos, além da idade, e afeta vários processos celulares. Várias mutações genéticas foram identificadas nos últimos anos (SNCA, GBA, LRRK2, PRKN) ${ }^{4}$. Os corpúsculos de Lewy, inclusões neuronais constituídas principalmente por agregados de proteína-a-sinucleína, são a principal alteração neuropatológica ${ }^{2,3}$.

O diagnóstico da DP é clínico, baseado nas manifestações motoras. Embora exames de imagem (TC de crânio, RM de crânio), e de imagem molecular (como o estudo do transportador de dopamina no estriado) possam ajudar a corroborar o diagnóstico, descartando outras etiologias para a síndrome parkinsoniana, não há um teste ou exame específico que conclua o diagnóstico de DP esporádica ${ }^{1,2}$.

Até o momento, não há cura para a doença, e o tratamento envolve medidas farmacológicas, principalmente preparações com levodopa, associadas ou não com outras medicações, além de outras medidas complementares, como fisioterapia, terapia ocupacional e fonoterapia. A bradicinesia e a rigidez melhoram bastante com a levodopa, mas o tremor geralmente apresenta pouca melhora ${ }^{3}$.

Mais recentemente, para os pacientes com tremor farmacorresistente, que apresentam piora significativa dos sintomas nos intervalos entre as doses de medicação ("wearing off"), e naqueles com discinesias ${ }^{3}$, modalidade de tratamento cirúrgico com implantação de estimulador cerebral profundo (deep brain stimulation - DBS), nos núcleos da base (NB), mais especificamente, no núcleo 
subtalâmico (NST) e/ou globo pálido interno (GPi; para tratamento da rigidez e da bradicinesia), ou tálamo (para tratamento do tremor) com estímulos a altas frequências (acima de $100 \mathrm{~Hz}$ ), de maneira crônica ${ }^{5}$, tem apresentado bons resultados terapêuticos a curto e a médio prazo, porém, sem evitar a progressão da doença. Os principais efeitos colaterais deste tratamento incluem aqueles relacionados ao ato operatório em si, piora da função cognitiva, sintomas psiquiátricos, e alterações oculares e da fala. Além disso, os sinais que não respondem à levodopa, como dificuldade para iniciar o movimento, sinais motores axiais (como o anterocórmio) e desequilíbrio, também não apresentam melhora significativa ${ }^{1}$.

\section{Modelos de disfunções dos núcleos da base e os distúrbios de movimento}

O modelo canônico de disfunção dos núcleos da base (NB) propõe que alterações na taxa de disparos neuronais são a base funcional para os distúrbios do movimento.

Projeções excitatórias vindas do córtex inervam o estriado e o NST (via super direta). O estriado contém uma população de células inibitórias, que se projetam para outros núcleos inibitórios, o GPi (via direta) e o globo pálido externo (GPe, via indireta). O GPe é reciprocamente conectado ao NST, que é excitatório. O NST emite projeções ao GPi, que por sua vez envia projeções ao tálamo. O tálamo, que excita o córtex, é então inibido pelo GPi. 
Especificamente na DP, ocorreria uma diminuição da atividade na via direta, que é inibitória. Em paralelo, há um aumento de atividade na chamada via indireta, que é "não inibitória" (inibe a via direta, que é inibitória). O efeito deste desbalanço, há um aumento a taxa de disparo dos neurônios inibitórios no globo pálido interno, com eferências para estruturas pré-motoras importantes, como o tálamo. A inibição dos centros pré-motores explica os sintomas de acinesia/bradicinesia, porém, não explica tremor e rigidez. Modelos animais mostraram que o aumento dos disparos no GPi é sutil, em DP experimental ${ }^{6,7}$.

Outros estudos também questionam esse modelo, uma vez que muitos neurônios dos NB têm propriedades de marca-passo intrínseco, disparando mesmo na ausência de conectividade $^{8}$. Além disso, não é possível explicar como tanto as lesões ablativas quanto a neuroestimulação do GPi, de maneira paradoxal, melhoram as discinesias, sem prejuízo das funções motoras ${ }^{9}$.

Basicamente, há duas formas de intervenção cirúrgica que melhoram os sintomas motores na DP: lesão do GPi ou NST, e estimulação destes mesmos locais com alta frequência através do DBS. Lesões do GPi deveriam abolir as aferências dos NB para o córtex motor, piorando o desempenho motor voluntário, mas, na DP ocorre justamente o contrário. A similaridade entre os efeitos de ablação e de estimulação a altas frequências poderia sugerir que estes estímulos fariam uma lesão virtual, bloqueando a função deste núcleo. Porém, na DP, os neurônios do GPi 
humano disparam em frequências entre 85 e $140 \mathrm{~Hz}$, sugerindo que é mais provável que haja um alinhamento dos elementos neurais com estímulos de alta frequência do que o bloqueio do funcionamento destes neurônios ${ }^{9}$.

Através de estudos neurofisiológicos em humanos e em modelos animais, Brown et al. propuseram um "modelo oscilatório" dos NB para explicar a interação dos diferentes ritmos oscilatórios da alça NB-córtex com os movimentos ${ }^{9,10}$. A premissa é que a atividade dos NB na DP envolve sincronização anormal, e que é preferível nenhuma atividade eferente palidal do que eferências com ruídos ${ }^{9}$. Neste modelo, atividades sincronizadas nas faixas entre 5 e $30 \mathrm{~Hz}$ antagonizam $\circ$ processamento motor (atividade anticinética), sendo necessária uma disrupção desta sincronização para que haja o movimento voluntário. De forma antagônica, oscilações na faixa gama encontram-se aumentadas, tanto nos NB quanto no córtex motor, concomitantemente aos movimentos voluntários, sendo "procinéticas"7,9,11. Em situações não fisiológicas, como no caso da depleção de dopamina, tais oscilações provavelmente envolvem uma população maior de neurônios disparando na mesma frequência, gerando uma atividade oscilatória patologicamente exagerada (Figura 1$)^{7,9}$. Deste modo, as oscilações de potenciais de campo local (local field potentials, LFP) que são gerados pelo DBS poderiam funcionar como filtros da transmissão estriatal, tanto nos impulsos de entrada quanto nos de saída?. 
Figura 1. Figura esquemática dos modelos canônico e oscilatório na doença de Parkinson.

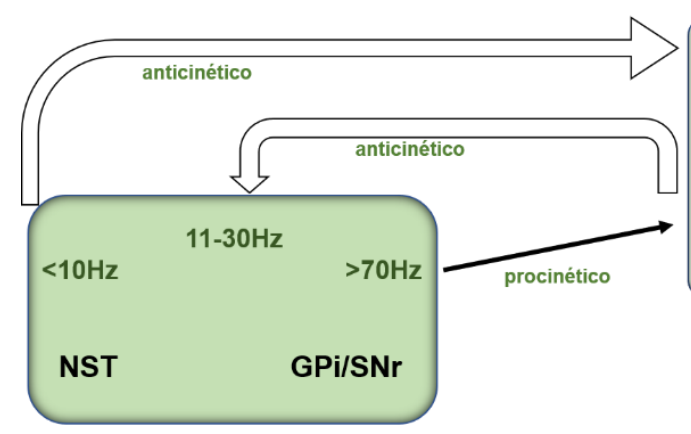

MODELO OSCILATÓRIO

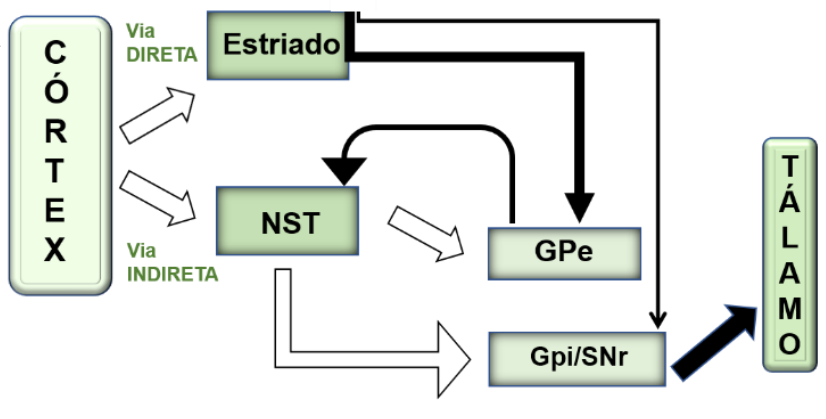

MODELO CANÔNICO

À direita, modelo canônico. Mudanças na atividade neural em decorrência da perda de dopamina estriatal são indicadas pelas setas largas (aumento) e estreitas (diminuição). Na doença de Parkinson, a atividade na via direta, com eferências do estriado para o globo pálido interno (GPi) e para a substância negra pars reticulata (SNpr) é diminuída, enquanto a atividade da via indireta, que passa pelo globo pálido externo (GPe) e núcleo subtalâmico (NST), encontra-se aumentada, levando ao aumento da taxa de disparos eferentes. Desta maneira, os centros premotores, como o tálamo, são inibidos, facilitando as eferências corticoespinhais. Via glutamatérgica (setas sem preenchimento): excitatória; via gabaérgica (setas preenchidas): inibitória. À esquerda, modelo oscilatório. Na ausência de dopamina no estriado, oscilações patológicas são iniciadas ou realçadas nos núcleos da base, as quais modulam os ritmos de outras regiões/núcleos (setas). Frequências abaixo de $10 \mathrm{~Hz}$ se iniciam nos núcleos da base e envolvem o córtex motor produzindo efeito anticinético. O NST é modulado por oscilações do córtex na faixa beta $(11-30 \mathrm{~Hz})$, que também são consideradas anticinéticas (setas largas sem preenchimento). Oscilações na faixa gama $(>70 \mathrm{~Hz})$, que facilitam os movimentos voluntários (procinéticas), são ausentes ou suprimidas (setas estreitas preenchidas).

\section{Neurofisiologia na DP}

As estruturas corticais e subcorticais do cérebro interagem através de oscilações neurais, as quais são classicamente divididas de acordo com a frequência em que ocorrem, nas faixas delta $(>4 \mathrm{~Hz})$, teta $(4-7 \mathrm{~Hz})$, alfa (8$13 \mathrm{~Hz})$, beta $(14-30 \mathrm{~Hz})$, e gama $(>30-100 \mathrm{~Hz})$. A disfunção patológica destas redes oscilatórias pode levara vários distúrbios neurológicos, entre estes, a DP. É possível registrar esta atividade oscilatória através de estudos com 
eletrencefalograma $(E E G)^{12}$, magnetoencefalografia $(M E G)^{13}$ e LFP ${ }^{10}$, estes últimos na avaliação neurofisiológica intraoperatória, através dos microeletrodos, ou no pósoperatório imediato, sendo o sinal captado pelos eletrodos implantados para a DBS, externalizados, antes de serem conectados com o gerador de pulso.

No sistema motor humano, em condições fisiológicas, um ritmo na frequência beta é registrado no córtex somatomotor, no sistema cerebelar e nos NB, modulado de acordo com a tarefa motora a ser realizada ou não ${ }^{5}$. As oscilações na faixa beta são consideradas um ritmo intrínseco que manteria as redes motoras em estado de repouso, com decremento em situações de movimento, nas quais aumentariam as oscilações gama ${ }^{14}$, estas últimas relacionadas ao estado de alerta, vigor motor e motivação ${ }^{15}$. É possível que o nível de atividade beta cortical no sistema motor seja inversamente proporcional à probabilidade de que uma nova ação voluntária se faça necessária, tanto para ser processada quanto executada. A atividade beta, ou sua supressão, determinaria a "prontidão motora"16.

Estudos dos ritmos do córtex sensitivomotor e dos NB na DP mostram que há coerência de atividade neurofisiológica nestes substratos ${ }^{17,18}$. É possível que a deficiência de dopamina nos NB esteja associada a alterações nos padrões de disparo na alça NB-córtex, nas vias direta e indireta, além de mudanças neurofisiológicas nas projeções tálamo-corticais, levando à disfunção do córtex motor. 
Dados crescentes sugerem que as oscilações anormais tenham um papel importante na $\mathrm{DP}^{9,10}$, sendo descrito um aumento de ritmos na faixa beta, tanto em pacientes quanto em modelos experimentais de DP, com concomitante decremento da faixa gama, em regiões diferentes dos NB (estriado, NST, GPi), e em áreas motoras corticais ${ }^{9,19}$. Estas alterações se correlacionam com o grau de bradicinesia e rigide ${ }^{11}$. Um estudo com pacientes implantados com DBS no NST mostrou que durante estímulos temporários com baixas frequências $(5-25 \mathrm{~Hz})$, há piora da bradicinesia ${ }^{20}$.

Porém, não é possível descartar que estas oscilações anormais tenham sua origem no próprio córtex ${ }^{18,21}$. Há evidências favoráveis ao entendimento de que os NB apresentam oscilações em certas frequências as quais são derivadas do córtex motor, tanto com estudos de microrregistro quanto por LFP. O significado funcional destas oscilações ainda não está completamente elucidado7. Apesar de a hipersincronia beta nas áreas corticais não ser crucial para a ocorrência de bradicinesia e rigidez, tal atividade é importante porque os impulsos corticais para os NB, sob circunstâncias de baixos níveis de dopamina, conseguem penetrá-los, amplificando os impulsos oscilatórios nesta frequência ${ }^{9}$. Então, uma estratégia terapêutica possível seria suprimir esta atividade cortical, estimulando vias aferentes ao córtex, ou o córtex propriamente dito 22,23 . 


\section{Dopamina e hipersincronia na faixa beta}

O nível de atividade beta nos NB pode ser alterado pela manipulação de dopamina e seus receptores. Registros de LFP com eletrodos de DBS mostram oscilações na frequência beta no GPi e no NST após a interrupção da reposição de levodopa, as quais diminuem com o retorno da medicação ${ }^{19}$.

Um estudo mostrou que os neurônios dopaminérgicos da substância negra mesencefálica operam de modo tônico e fásico, em condições fisiológicas, isto é, disparam a baixas frequências regularmente (modo tônico), porém, em resposta a um estímulo ambiental, por exemplo, disparam um surto de potenciais de ação de alta frequência (modo fásico). Estes surtos induzem uma liberação de grande quantidade de dopamina para o meio extracelular, em comparação à atividade tônica, e isto é amplificado em decorrência da integração que ocorre entre populações neurais, seja por acoplamento elétrico dos surtos fásicos, seja por impulsos aferentes comuns, relacionados a dado estímulo ${ }^{24}$. Um evento significativo no ambiente faz aumentar o nível de dopamina extracelular, de maneira que este nível nas vias dopaminérgicas seja suficiente para garantir que uma ação motora, sendo este aumento proporcional à probabilidade de que a mesma seja necessária. A supressão da sincronia na faixa beta poderia determinar o comportamento diante dos estímulos externos ${ }^{16}$. Nos pacientes com DP, há menos dopamina présináptica para ser liberada, tornando os disparos tônicos menos eficazes, de modo que a rede dopaminérgica funcione 
em um nível abaixo do que seria necessário. Isto se traduz por uma hipersincronia beta cortical, que ainda pode ser suprimida em resposta aos estímulos externos durante certo tempo. Os movimentos são lentos e com tempo de reação prolongado. É possível que o tratamento com levodopa restaure esta atividade dopaminérgica nas vias para a sua faixa fisiológica, atenuando a atividade beta e melhorando a execução dos movimentos voluntários.

$O$ entendimento dos fatores que influenciam estas oscilações corticais e subcorticais, como estado de reposição de levodopa, planejamento e execução de movimentos voluntários, além do estado clínico dos pacientes com DP, pode ajudar no entendimento da fisiopatologia da doença e nas estratégias terapêuticas, baseando-se nos achados eletrofisiológicos ${ }^{7,21}$.

\section{Modulação das oscilações por estimulador cerebral profundo (DBS) na doença de Parkinson}

Embora tenha sido proposto que DBS funciona interrompendo as oscilações patológicas ${ }^{25}$, estudos falharam em demonstrar essa resposta 26 .

Quando há correlação entre as fases de diferentes frequências que estão sendo geradas ao mesmo tempo, na mesma direção, ocorre ganho de amplitude, e isto é chamado acoplamento de fase-amplitude (AFA). De outro modo, quando as frequências estão fora de fase, ou em direções opostas, vai haver cancelamento de fase, com perda de amplitude. Nos pacientes com DP, ocorre um AFA 
entre oscilações na faixa beta de baixa frequência e aquelas na faixa gama, no córtex motor primário. ADBS inserida no NST reduziu este AFA, o que se associou à redução dos sinais parkinsonianos. É possível que índices de EEG-AFA sejam biomarcadores da eficiência ou não da modulação por DBS/levodopa sobre o córtex motor, de modo que os pacientes sem levodopa/sem DBS apresentem um índice alto, o qual tenderia à normalização durante a reposição da levodopa/com DBS ${ }^{27}$.

Recentemente, oscilações sintonizadas na frequência gama foram relatadas durante, ou imediatamente, após o implante de eletrodo para DBS no NST, na ausência de levodopa, em coerência com o registro de EEG. A resposta a diferentes frequências de estimulação e os efeitos prolongados após as mesmas sugerem que as oscilações são induzidas quando há ausência de levodopa. É possível que ocorra uma reconfiguração dos circuitos dos NB viabilizando estas oscilações. A presença de mudanças lentas nas frequências rápidas durante os bloqueios consecutivos de DBS sugere que esta reconfiguração de circuitos pode por si só evoluir no tempo 28 .

É possível que a levodopa tenha mais influência na modulação destes ritmos que o DBS por si só ${ }^{26}$. É preciso mais estudos para melhorar este entendimento. 


\section{Sincronização/dessincronização evento-relacionada no núcleo subtalâmico e cognição}

O NST exerce um papel importante na cognição ${ }^{29}$. Alterações oscilatórias nos sinais de EEG podem decorrer da sincronização ou dessincronização evento-relacionada (event related syncronization/desynchronization, ERS/D) de alguns dos componentes da frequência. ERS/D são fenômenos tempo relacionados, mas não fase relacionados, e são analisados como mudanças de potência (valores de amplitudes quadradas). A dessincronização representa $O$ decremento de potência, e a sincronização, o aumento. A atividade beta no NST é dessincronizada antes de um movimento voluntário espontâneo, bem como antes de um movimento evocado, e esta dessincronização se correlaciona com a força motora. De maneira antagônica, o aumento da potência beta é relacionado à capacidade de interromper uma ação motora. Porém, a reatividade beta é influenciada também por fatores contextuais, como recompensa, ou complexidade cognitiva.

Sincronização evento relacionada na frequência alfa no NST foi associada a tarefas que necessitam de atenção, tomada de decisões conflituais, e uma diminuição focal na potência alfa foi relacionada a visualização de figuras que continham significado emocional, em detrimento daquelas neutras. A diminuição desta potência alfa foi menor com estímulos agradáveis, e maior para aqueles desagradáveis, nos pacientes com DP e depressão concomitante. O aumento 
de atividade teta-alfa é associado com distúrbios no controle de impulso e jogo patológico na DP30.

\section{CONCLUSÃO}

A DP é uma doença neurodegenerativa muito prevalente na população, e o diagnóstico é essencialmente clínico. As modalidades de tratamento disponíveis não mudam a evolução da doença. O tratamento com levodopa melhora os sintomas motores, e a implantação de DBS no NST/GPi, tem sido indicada principalmente para aqueles pacientes que apresentam discinesia tardia relacionada ao uso de levodopa/wearing off.

O avanço das técnicas neurofisiológicas, tanto pela incorporação de novas tecnologias de registro da atividade neural (MEG), e do processamento de sinal (análise espectral, AFA, ERS/D), quanto a análise de coerência entre os sinais neurofisiológicos LFP dos NB e os de EEG, pode trazer novos entendimentos quanto à fisiopatogênese da DP, bem como novas propostas terapêuticas a partir deste entendimento. A evolução tecnológica dos equipamentos de DBS pode tornar este tratamento ainda mais eficiente. Novas modalidades de estímulos corticais e novos alvos terapêuticos podem ser desenvolvidos para pacientes que apresentam contraindicações clínicas, ou que seriam maus respondedores ao $\mathrm{DBS}^{23}$. Neste cenário tão desafiador, estudos neurofisiológicos continuam sendo necessários, 
tanto para verificar as novas descobertas, quanto para trazer inovações.

\section{REFERÊNCIAS}

1.Balestrino R, Schapira A. Parkinson disease. Eur J Neurol 2020;27:27-42. https://doi.org/10.1111/ene.14108

2.Schapira AHV, Chaudhuri KR, Jenner P. Non-motor features of Parkinson disease. Nat Rev Neurosci 2017;18:435-50. https://doi.org/10.1038/nrn.2017.62

3.Armstrong MJ, Okun MS. Diagnosis and treatment of Parkinson's disease: a review. JAMA 2020;323:548-60. https://doi.org/10.1001/jama.2019.22360

4. Kalia LV, Lang AE. Parkinson's disease. Lancet 2015;386:896-912. https://doi.org/10.1016/S0140-6736(14)61393-3

5.Brown P. Abnormal oscillatory in the motor system leads to impaired movement. Curr Opin Neurobiol 2007;17:656-64. https://doi.org/10.1016/j.conb.2007.12.001

6. Levy R, Dostrovsky JO, Lang AE, Sime E, Hutchison WD, Lozano AM. Effects of apomorphine on subthalamic nucleus and globus pallidus internus neurons in patients with Parkinson's disease. J Neurophysiol 2001;86:249-60. https://doi.org/10.1152/jn.2001.86.1.249

7. Hutchison WD, Dostrovsky JO, Walters JR, Courtemanche R, Boraud $\mathrm{T}$, Goldberg J, et al. Neuronal oscillations in the basal ganglia and movement disorders: evidence from whole animal and human recordings. J Neurosci 2004;24:9240-3. https://doi.org/10.1523/JNEUROSCI.3366-04.2004

8. Bevan MD, Magill PJ, Terman D, Bolam JP, Wilson CJ. Move to the rhythm: oscillations in the subthalamic nucleus-external globus pallidus network. Trends Neurosci 2002;25:525-31. https://doi.org/10.1016/s0166-2236(02)02235-x

9. Brown P. Oscillatory nature of human basal ganglia activity: relationship to the pathophysiology of Parkinson's disease. Mov Disord 2003;18:357-63. https://doi.org/10.1002/mds.10358

10.Brown P, Williams D. Basal ganglia local field potential activity: character and functional significance in the human. Clin Neurophysiol 2005;116:2510-9. https://doi.org/10.1016/j.clinph.2005.05.009

11.Kühn AA, Kempf F, Brücke C, Gaynor Doyle L, Martinez-Torres I, Pogosyan $A$, et al. High-frequency stimulation of the subthalamic nucleus suppresses oscillatory beta activity in patients with Parkinson's disease in parallel with improvement in motor performance. J Neurosci 2008;28:6165-73. https://doi.org/10.1523/JNEUROSCI.0282$\underline{08.2008}$

12. Marsden JF, Limousin-Dowsey $\mathrm{P}$, Ashby $\mathrm{P}$, Pollak $\mathrm{P}$, Brown $\mathrm{P}$. Subthalamic nucleus, sensorimotor cortex and muscle 
interrelationships in Parkinson's disease. Brain 2001;124:378-88. https://doi.org/10.1093/brain/124.2.378

13.Boon I, Geraedts VJ, Hillebrand A, Tannemaat MR, Contarino MF, Stam CJ, et al. A systematic review of MEG-based studies in Parkinson's disease: The motor system and beyond. Hum Brain Mapp 2019;40:2827-48. https://doi.org/10.1002/hbm.24562

14.Engel AK, Fries P. Beta-band oscillations-signalling the status quo? Curr Opin Neurobiol 2010;20:156-65. https://doi.org/10.1016/j.conb.2010.02.015

15.Mazzoni P, Hristova A, Krakauer JW. Why don't we move faster? Parkinson's disease, movement vigor, and implicit motivation. J Neurosci 2007;27:7105-16.

https://doi.org/10.1523/JNEUROSCI.0264-07.2007

16.Jenkinson N, Brown P. New insights into the relationship between dopamine, beta oscillations and motor function. Trends Neurosci 2011;34:611-8. https://doi.org/10.1016/j.tins.2011.09.003

17. Hirschmann J, Özkurt TE, Butz M, Homburger M, Elben S, Hartmann $\mathrm{CJ}$, et al. Distinct oscillatory STN-cortical loops revealed by simultaneous MEG and local field potential recordings in patients with Parkinson's disease. Neuroimage 2011;55:1159-68. https://doi.org/10.1016/j.neuroimage.2010.11.063

18. Litvak V, Jha A, Eusebio A, Oostenveld R, Foltynie T, Limousin $P$, et al. Resting oscillatory cortico-subthalamic connectivity in patients with Parkinson's disease. Brain 2011;134:359-74. https://doi.org/10.1093/brain/awq332

19. Hammond C, Bergman $\mathrm{H}$, Brown P. Pathological synchronization in Parkinson's disease: networks, models and treatments. Trends Neurosci 2007; 30:357-64. https://doi.org/10.1016/j.tins.2007.05.004

20.Fogelson N, Kühn AA, Silberstein $P$, Limousin PD, Hariz $M$, Trottenberg $\mathrm{T}$, et al. Frequency dependent effects of subthalamic nucleus stimulation in Parkinson's disease. Neurosci Letters 2005;382:5-9. https://doi.org/10.1016/j.neulet.2005.02.050

21.Udupa K, Chen R. Chapter 10. Motor cortical circuits in Parkinson disease and dystonia. Handbook Clin Neurol 2019;161:167-86. https://doi.org/10.1016/B978-0-444-64142-7.00047-3

22.Eusebio A, Thevathasan W, Doyle Gaynor L, Pogosyan A, Bye E, Foltynie $T$, et al. Deep brain stimulation can suppress pathological synchronisation in parkinsonian patients. J Neurol Neurosurg Psychiatry

https://doi.org/10.1136/jnnp.2010.217489

23. Underwood CF, Parr-Brownlie LC. Primary motor cortex in Parkinson's disease: functional changes and opportunities for neurostimulation. Neurobiol Dis 2021;147:1-15. https://doi.org/10.1016/j.nbd.2020.105159

24. Hyland BI, Reynolds JN, Hay J, Perk CG, Miller R. Firing modes of midbrain dopamine cells in the freely moving rat. Neurosci 2002;114:475-92. https://doi.org/10.1016/s0306-4522(02)00267-1 
25.Müller EJ, Robinson PA. Suppression of Parkinsonian beta oscillations by deep brain stimulation: determination of effective protocols. Front Comput Neurosci 2018;12:1-16. https://doi.org/10.3389/fncom.2018.00098

26.Giannicola G, Marceglia S, Rossi L, Mrakic-Sposta S, Rampini P, Tamma $F$, et al. The effects of levodopa and ongoing deep brain stimulation on subtalamic beta oscillations in Parkinson's disease. Exp Neurol 2010;226:120-7. https://doi.org/10.1016/j.expneurol.2010.08.011

27.Swann NC, de Hemptinne C, Aron AR, Ostrem JL, Knight RT, Starr PA. Elevated synchrony in Parkinson disease detected with electroencephalography. Ann Neurol 2015;78:742-50. https://doi.org/10.1002/ana.24507

28. Wiest C, Tinkhauser G, Pogosyan A, He GS, Baig F, Morgante F, et al. Subthalamic deep brain stimulation induces finely-tuned gamma oscillations in the absence of levodopa. Neurobiol Dis 2021, in press. https://doi.org/10.1016/j.nbd.2021.105287

29. Weintraub DB, Zaghloul KA. The role of the subthalamic nucleus in cognition. Rev Neurosci 2013;24:125-38. https://doi.org/10.1515/revneuro-2012-0075

30.Rosa M, Fumagalli M, Giannicola G, Marceglia S, Lucchiari C, Servello $D$, et al. Pathological gambling in Parkinson's disease: subthalamic oscillations during economics decisions. Mov Disord 2013;28:1644-52. https://doi.org/10.1002/mds.25427 